\title{
QUANTUM ORIGIN OF SPONTANEOUS COMPACTIFICATION
}

\author{
Claudio A. Orzalesj \\ CERN -- Geneva \\ and
}

Sezione Teorica, Dipartimento di Fisica dell' Università 43100 Parma, and INFN, Sezione di Milano, Milano, Italy

and

Giovanni Venturi

Dipartimento di Fisica dell'Università and INFN, Bologna Italy

\section{ABSTRACT}

The possible quantum dynamical origin of spin torsion spontaneous compactification is investigated. The Yang-Mills coupling constant is calculated dynamically in a simple model, in terms of a finite cut-off. 

A basic ingredient of the modern multidimensional unified theories 1)-6) is the occurrence of spontaneous compactification 4$), 6$ ). The background geometry, which describes "empty space" in $\mathrm{D}=4+\mathrm{K}$ dimensions, is not trivial but rather corresponds to $\mathrm{M}_{4}^{\mathrm{O}} \times \mathrm{V}^{\mathrm{K}}$, where $\mathrm{M}_{4}^{\circ}$ is (Minkowski) 4 and $\mathrm{V}^{\mathrm{K}} \simeq \mathrm{G} / \mathrm{H}$, where $G$ is a non-Abelian compact group and $H$ a subgroup. As is well known, if spontaneous compactification occurs then the zero modes of the metric * $\gamma_{\mathrm{MN}}$ on the background $\mathrm{M}_{4}^{\mathrm{O}} \times \mathrm{V}^{\mathrm{K}}$ yield, in four dimensions, one graviton coupled to the Yang-Mills fields of $G$ and possibly to generalized 3) Jordan-Thiry 2) scalar fields.

As explained in Ref. 6), there are two basic mechanisms which can lead to a non-trivial spontaneous compactification :

(A) a suitable energy momentum condensate in the quantum ground state ( $q$ vacuum $|0\rangle)$, and/or

(B) a suitable spin torsion condensate in the $q$ vacuum.

Mechanism (A) can be effective already at the classical level, through suitable q vacuum expectation values of bosonic fields ${ }^{* *}$ ). On the othex hand, (B) is typically a quantum mechanism because, in Einstein-Cartan (EC) theories, the spin density leading to torsion is due to local fermion bilinears 7). The two mechanisms, (A) and (B), could also operate together : a remarkable example is given by $D=11$ supergravity ${ }^{8}$ ), where a phase with a "bosonic" compactification $M_{4}^{\Lambda} \times V^{7}$, see Ref. 9), over anti-de Sitter space-time $M_{4}^{\Lambda}$, can be followed ${ }^{10)}$ by a phase with spin-torsion compactification over $\mathrm{M}_{4}^{0} \times \mathrm{V}^{7}$. We recall that mechanism (B) leads to a vanishing cosmological constant 11 ) and to fermionic zero mass modes in four dimensions 6 ).

By considering the $q$ vacuum expectation values of the EC equations, a further comparison is possible between mechanisms (A) and (B), if one separates the Riemannian Einstein tensor from the piece due to torsion : with (A) one assumes that the torsion contribution vanishes but that the Riemannian Einstein tensor and the Riemannian energy momentum tensor are not trivial in the $q$ vacuum. With $(B)$, the torsion contribution is not vanishing but the

*) Capital Latin indices run from 1 to $D$; Greek from 1 to 4 and refer to $\mathrm{MO}$; lower-case Latin run from 5 to $\mathrm{D}=4+\mathrm{K}$ and refer to $\mathrm{V}$. "Early" indices are anholonomic, "late" indices are holonomic. We write $\mathrm{X}=(\mathrm{x}, \mathrm{y})$ for $\mathrm{X} \in \mathrm{M}_{4}^{\mathrm{O}} \times \mathrm{V}^{\mathrm{K}}, \mathrm{x} \in \mathrm{M}_{4}^{\mathrm{O}}, \mathrm{y} \in \mathrm{V}$.

**) However, known classical models 4) with the flat base space Mo require the fine tuning of a cosmological constant and Yang-Milis fields in D dimensions. Sugh fields defeat the purpose of a Kaluza-Klein unification 1),6). 
full Einstein-Cartan tensor and the canonical energy-momentum tensor are both vanishing in the $q$ vacuum. Therefore, in approaches based on ( $A$ ), one is neglecting the possibility of a non-trivial $q$ vacuum spin energy, while with (B) it is assumed that $a_{*} q$ vacuum spin energy is crucial to make the background geometry stable ${ }^{*}$ ).

The occurrence of non-trivial vacuum expectation values of some components of the spin density is akin to the phenomenon of dynamical symmetry breaking. In fact, the spin-spin contact interaction in the EC theory is similar to the Nambu-Jona Lasinio ( $N-J L$ ) chiral coupling ${ }^{13}$, and the functional methods previously used ${ }^{14}$ ) for the N-JI model are easily generalized $^{12)}$, so as to deal with torsion dynamics in the quantized EC theory.

In the present paper, such analogies are pursued further, in order to draw some indications on the possible dynamical origin of spin torsion spontaneous compactification. We seek a self-consistent approximation for the spin-spin contact interaction, and we formulate a generalized N-JL consistency condition. In essence, this is an "anti-gap" equation : we require that the lowest bare fermion mass, which inevitably occurs in the Riemannian case 15),6), is driven down to zero physical mass by the self-energy due to torsion **). In a simple seven-dimensional model, we show that this leads precisely to the parallelizing torsion on $\mathrm{s}^{3}$, as advocated in Ref. 6). The consistency of the approach is then checked, as in Refs. 6),11), by showing that $M_{4}^{0} \times S^{3}$ is indeed a solution of the background $E C$ equations.

As in Ref. 12), we isolate the "Riemannian" gravity effects and we deal with the path integral

$$
Z\left(E^{A}\right)=\int D \underline{K} D \bar{\Psi} D \Psi \exp i\left(A_{k}+A_{D}\right)
$$

where $\mathrm{E}^{\mathrm{A}}=\mathrm{E}^{\mathrm{A}} \mathrm{dX}$ is an external vielbein (with $\mathrm{A}, \mathrm{M}=1, \ldots, \mathrm{D}$ and with metric $\left.\gamma_{M N}=\eta_{A B} E^{A} E_{N}^{B^{M}}\right), \quad K_{A B C}$ is the contorsion, and, with $\stackrel{\tau}{\sim}=E^{1} \ldots \ldots E^{D}$,

*) As emphasized in Ref. 12), the conventional arguments for studying the stability of background geometries are wholly inadequate when the spin density can develop a non-trivial vacuum expectation value.

**) There is a four-dimensional analogue to this screening of the bare mass: if one introduces a static background torsion along the $z$ axis, then the Fermi sphere becomes depleted along the other two axes 16). Similarly, a background torsion in the internal space $\mathrm{V}^{\mathrm{K}}$ lowers the fermion masses as seen on $\mathrm{M}_{4}^{\circ}$. The resulting change in the zerp-point mion masses as seen on ${ }_{4}$. The resulting change in the 17$), 18$ ) Casimir effect. 


$$
\begin{aligned}
& A_{K}=-\frac{1}{2 \hat{x}} \int \underline{K} \cdot \underset{K}{\tau}, \\
& A_{D}=-\frac{1}{2} \int \bar{\Psi}\left\{\nabla_{A}(\omega), \Gamma^{A}\right\} \Psi \tau .
\end{aligned}
$$

$\mathcal{A}_{\mathrm{K}}$ and $\mathfrak{A}_{\mathrm{D}}$ are the contributions to the EC action due to the contorsion and to a Dirac field $\Psi$ which is "massless" in $D$ dimensions. As usual

$$
\nabla_{A}(\omega)=\nabla_{A}(\stackrel{0}{\omega})-\frac{1}{4} K_{A B C} \Gamma^{B C}
$$

where $\stackrel{\circ}{A B C}_{A B C}$ is the Levi-Civita connection of $\mathbb{E}^{\mathrm{A}}$, and $\hat{x}$ is the $D$ dimensional analogue of Newton's constant $x$ (we shall latex set $\hat{\mu}=u \mathrm{~V}$, where $\mathrm{V}$ is the volume of $\mathrm{V}^{\mathrm{K}}$ ).

We consider a simple model in $\mathrm{D}=7$, and we choose

$$
\left(E^{A}\right)=\left(e^{\alpha}, e^{a}\right): \quad e_{M}^{\alpha}=e_{\mu}^{\alpha}(x) \delta_{M}^{\mu}, e_{M}^{a}=e_{m}^{a}(y) \delta_{M}^{m},
$$

where $\alpha, \mu=1, \ldots, 4$ and $a, m=5,6,7$, with co-ordinates $\left(x^{\mathbb{M}}\right)=\left(x^{\mu}, y^{m}\right)$. It will be assumed that $e^{a}(y) d y^{m}$ is a three-bein on $s^{3}$; by identifying $s^{3}$ with SU(2), we choose $e^{a}$ to be the left-invariant Maurer-Cartan forms on $\mathrm{SU}(2)$. We shall later verify that the choice (5) with $e_{\mu}^{\alpha}=\delta_{\mu}^{\alpha}$, which corresponds to $\mathrm{M}_{4}^{0} \times \mathrm{S}^{3}$, is indeed a solution of the background $\mathrm{EC}$ equations, after a suitable background torsion has been generated.

The Cartan field equation gives

$$
K_{A B C}=\frac{\hat{x}}{4} \bar{\Psi} \Gamma_{A B C} \Psi=K_{[A B C]}=\frac{1}{2} T_{A B C} \text {, }
$$

where $\mathrm{T}_{\mathrm{ABC}}$ is the torsion. In particular, let us consider the "internal" components $\mathrm{K}_{a b c}, a, b, c=5,6,7$ : these are the only components of $\mathrm{K}_{\mathrm{ABC}}$ which, on the background $e_{\mu}^{\alpha}=\delta_{\mu}^{\alpha}$, can develop a $q$ vacuum expectation value

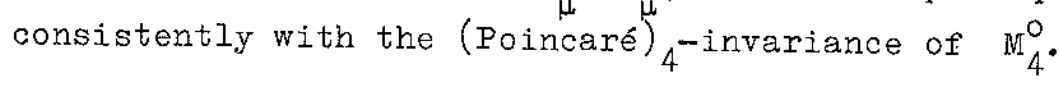

$$
\begin{aligned}
& \text { Now, if one expands } K_{a b c}(x, y) \text { in harmonics over } s^{3} \text {, } \\
& K_{a b c}(x, y)=\sum_{\lambda} K_{a b c}^{(\lambda)}(x) D^{(\lambda)}(y),
\end{aligned}
$$

it is interesting to consider the 0 mode $K_{a b c}^{(0)}$, which coxresponds to $\lambda=0$ and, when the left-invariant three-bein $e^{a}$ is used, to the singlet representation $D^{(0)}$ of $\mathrm{SU}(2)$. Notice that $\mathrm{K}_{a b c} \mathrm{e}^{\mathrm{a}} \mathrm{e}^{\mathrm{b}} \mathrm{e}^{\mathrm{c}}$ is a three-form on 
$\mathrm{SU}(2)$ and that, with the standard $\mathrm{SU}(2) \times \mathrm{SU}(2)$ invariant metric, only $\mathrm{K}_{\mathrm{abc}}^{(\mathrm{o})} \mathrm{e}^{\mathrm{a}} \wedge \mathrm{e}^{\mathrm{b}} \wedge \mathrm{e}^{\mathrm{c}}$ is $\mathrm{SU}(2) \times \mathrm{SU}(2)$ invariant. By a general theorem ${ }^{19)}, \mathrm{K}_{\mathrm{abc}}^{(0)} \times$ $e^{a}{ }_{a}{ }^{b}, e^{c}$ is the only harmonic three-form on $s^{3}: \Delta{ }^{(3)} \mathrm{K}_{a b c}^{(0)}=0$. Therefore, only $\mathrm{K}_{a b c}^{(0)}$ shall not inherit a mass term by dimensional reduction on $\mathrm{M}_{4}^{\circ}$, while $\mathrm{K}_{a b c}(\lambda), \lambda \neq 0$, will correspond to fields on $\mathrm{M}_{4}^{0}$ with a mass term induced by the $y$ dependence ${ }^{*}$ ).

Let us deal with $\mathrm{K}_{a b c}^{(0)}(\mathrm{x})$ and neglect the remaining contorsion contributions appearing in Eqs. $(1)-(3)$. Since $\mathrm{K}_{\mathrm{abc}}=\mathrm{K}_{[\mathrm{abc}]}$, we can write

$$
K_{a b c}^{(0)}=\varepsilon_{a b c} \chi(x) \text {, }
$$

where $x$ is a scalar field on $\mathbb{M}_{4}^{0}$. We are interested in the possibility that $X$ may develop a $q$ vacuum value,

$$
x=\hat{x}+x_{0} \quad, \quad x_{0} \equiv\langle 0|x| 0\rangle \neq 0 .
$$

Of course, when $e_{\mu}^{\alpha}=\delta_{\mu}^{\alpha}, x_{0}$ must be independent of $x \in \mathbb{M}_{4}^{0}$, to preserve the (Poincaré) 4 invariance of $|0\rangle$. Notice also that, by (8), the background value

$$
\left\langle K_{a b c}\right\rangle_{0}=\varepsilon_{a b c} \chi_{0},
$$

is consistent with the $\mathrm{SU}(2) \times \mathrm{SU}(2)$ symmetry of the background internal metric on $s^{3}$.

To simplify the discussion as much as possible, we concentrate on the dynamical effects of $\mathrm{K}_{\mathrm{abc}}^{(0)}$ and we deal with the following amputated action :

$$
\begin{aligned}
\bar{A} & =\bar{A}_{K}+\vec{A}_{D}, \\
\bar{A}_{K} & =-\frac{1}{2 \hat{x}} \int K_{a b c}^{(0)} K^{(0) a b c} e^{1} \wedge \ldots e^{7} \\
& =-\frac{3}{x} \int\left(\hat{x}+X_{0}\right)^{2} e^{1} \wedge \ldots \wedge e^{4},
\end{aligned}
$$
*) Notice that this does not imply that $\mathrm{K}_{\mathrm{abc}}^{(0)}$ is massless in four
dimensions, because it has a bare mass $\left(\sim \sim^{\left.-\frac{1}{2}\right)}\right.$ from Eq. $(2)$. 
$-5-$

$$
\bar{A}_{D}=-\int \bar{\Psi}\left(\not D(\dot{\omega})-\frac{1}{4} X \varepsilon_{a b c} \Gamma^{a b c}\right) \Psi e^{1} \wedge \ldots 1 e^{7},
$$

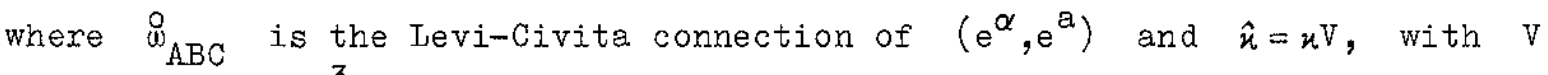
the volume of $\mathrm{s}^{3}$.

$$
\begin{aligned}
& \text { We now proceed as in Refs. 6) and 12), with the representation } \\
& \Gamma^{\alpha}=\hat{\gamma}^{\alpha} \otimes \mathbb{1}, \Gamma^{a}=\gamma^{5} \otimes \sigma^{a}, \quad \hat{\gamma}^{\alpha}=-i \gamma^{5} \gamma^{\alpha},
\end{aligned}
$$

and with the effective mass operators

6)

$$
\begin{aligned}
& \dot{M}_{\text {op }}=i\left(\sigma^{a} e_{a}-\frac{1}{4} \dot{\omega}_{a b c} \sigma^{a b c}\right)=i \sigma^{a} e_{a}-\frac{3}{4 \sqrt{2} L}, \\
& M_{o p}=\dot{M}_{o p}-\frac{i}{4}\left\langle K_{a b c}\right\rangle_{0} \sigma^{a b c}=\dot{M}_{o p}-\frac{3}{2} x_{0},
\end{aligned}
$$

where $I$ is the length of $S^{3}$ as defined in Ref. 6). As in Ref. 6), one can expand $\psi(x, y)$ into the eigenmodes of $M_{\text {op }}$. We call $\psi_{j}(x), y_{*}^{j}=0, \frac{1}{2}, 1, \ldots$, the physical Dirac field in four dimensions which corresponds ${ }^{*}$ to the hormanic associated to the eigenvalue $\mathbb{N}_{j}$ of $\left|\stackrel{\mathrm{M}}{o p}_{\text {op }}\right|$ and $\mathbb{M}_{j}$ of $\left|\mathbb{M}_{\text {op }}\right|$. By performing the integral over $S^{3}$, the action (15) is reduced to

$$
\bar{A}_{D}=-\sum_{j} \int \bar{\psi}_{j}\left(\not D^{(4)}(\dot{\omega})+M_{j}-\frac{3}{2} \hat{x}\right) \psi_{j} e^{1} \wedge \ldots \wedge e^{4},
$$

when re

$$
M_{j}=\dot{M}_{j}-\frac{3}{2} x_{0}
$$

and

$$
\not \not^{(4)}=\gamma^{\alpha}\left(e_{\alpha}-\frac{1}{4} \dot{\omega}_{[\alpha \beta \gamma]} \gamma^{\alpha \beta \gamma}\right. \text {. }
$$

Now, the path integral $Z\left(\mathrm{E}^{\mathrm{A}}\right)$ has been truncated to

*) The spectra of $M_{o p}$ are not positive-definite; this simply means that, for negative eigenvalues, $\psi_{j}$ is related by a Pauli-Guirsey transformation to the corresponding component of $\psi$. 
$-6-$

$$
\bar{Z}\left(e^{\alpha}, e^{a}\right)=\int D \hat{x} \prod_{j} D \bar{\psi}_{j} D \psi_{j} \exp i\left(\bar{A}_{K}+\bar{A}_{D}\right) .
$$

As in Ref. 12), we proceed by integrating over the fermions, so as to find the effective action for the torsion:

$$
\bar{Z}\left(e^{\alpha}, e^{a}\right)=N\left(e^{\alpha}, e^{a}\right) \int \partial \hat{x} \exp i \bar{A}_{K}^{e f f},
$$

where

$$
\begin{aligned}
& \vec{A}_{K}^{\text {eff }}=-\frac{3}{x} \int\left(\hat{x}+x_{0}\right)^{2} e^{1} \wedge \ldots \wedge e^{4}+\sum_{n=1}^{\infty} d_{j} u_{j}^{(n)}, \\
& u_{j}^{(n)}=\frac{i}{n} \operatorname{tr}\left(\frac{3}{2} S_{j} \hat{x}\right)^{n},
\end{aligned}
$$

with $a_{j}$ the multiplicity of the eigenvalue $\mathbb{M}_{j}$ and

$$
\begin{aligned}
& N\left(e^{\alpha}, e^{a}\right)=\exp t_{\imath} \log \left(-\sum_{j} d_{j} S_{j}^{-1}\right), \\
& S_{j}^{-1}=\gamma^{\alpha}\left(e_{\alpha}-\frac{1}{4} \dot{\omega}_{[\alpha \beta \gamma]} \gamma^{\beta \gamma}\right)+M_{j} .
\end{aligned}
$$

The term $n=1$ in (20) yields a contribution which is linear in $\hat{x}$. Since $\hat{x}$ has vanishing expectation value, the total contribution to $\overline{\mathfrak{A}}_{\mathrm{K}}^{\mathrm{eff}}$ which is linear in $\hat{x}$ must vanish, and we obtain the following generalization of the N-JI consistency condition :

$$
X_{0}=i \frac{x}{4} \sum_{j} d_{j} t_{r} S_{j} \text {. }
$$

Now, in a "realistic" multidimensional theory, we should: (i) set $e^{\alpha}{ }_{\mu}=\delta^{\alpha}{ }_{\mu}$, corresponding to the $\mathbb{M}_{4}^{0}$ background, and (ii) require that zero mass fermions occur in four dimensions, probably to be identified with loptons ${ }^{20)}$. We therefore demand that the gap $f_{0}$ be completely compensated by the induced torsion mass counter-term $\frac{3}{2} x_{0}$, with zero physical mass for the 
lowest mode $(\hat{j}=0)$. This fixes the magnitude of the internal length $\mathrm{L}$ in terms of the vacuum expectation value of the contorsion: with the notation of Ref. 6), section 3E, one obtains ${ }^{*}$ )

$$
L \dot{M}_{0}=\frac{3}{4 \sqrt{2}}=\frac{3}{2} x_{0} L .
$$

Going back to Eqs. (9) and (10), from the result $\left\langle 0\left|\mathrm{~K}_{\mathrm{abc}}\right| 0\right\rangle=\varepsilon_{a b c} / 2 \sqrt{2} \mathrm{I}$ and by comparison with Eqs. (3.6b), (3.43) and (3.44) of Ref. 6), we see that the background torsion is precisely the one ${ }^{*}$ which leads to vanishing Riemann-Cartan curvature on $\mathrm{s}^{3}$. It follows that the background connection has vanishing Einstein-Cartan tensor and that the background geometry is indeed a solution of the EC classical field equations ${ }^{*}$ ).

Next, we combine (25) with the self-consistency condition (24), where now the mass eigenvalues $M_{j}$ are the same as those calculated in Eq. (3.44) of of Ref. 6) :

$$
\begin{aligned}
& L M_{j}=c_{j}, j=0, \frac{1}{2}, 1, \ldots \\
& \sqrt{2} c_{j}=J+\frac{1}{2} \pm 1, J=\frac{1}{2}, 1, \ldots ; j=J \pm \frac{1}{2} ; \sqrt{2} c_{1 / 2}=3 .
\end{aligned}
$$

One obtains, setting $c_{j}^{\prime}=c_{j} d_{j}$,

$$
1=\frac{x}{\sqrt{2}} \sum_{j \neq 0} c_{j}^{\prime} \frac{i}{(2 \pi)^{4}} \int \frac{1}{p^{2}+M_{j}^{2}} d^{4} p .
$$

In complete analogy with what happens in the N-JL model, the consistency condition (28) is meaningless unless a cut-off $\left(p_{0}^{2}+p^{2}<M^{2}, j<T\right)$ is introduced, leading to

*) Since we are dealing with the absolute value of the lowest eigenvalue of $\mathrm{M}_{\mathrm{op}}$, there is a sign ambiguity in (25); we arbitrarily chose the positive sign, which corresponds to the $(+)$ Cartan-Schouten connection on $\mathrm{SU}(2)$. The alternative choice leads to the $(-)$ connection, and corresponds to the interchange between the left- and right-invariant Maurer-Caxtan forms on SU(2), see Ref. 6), Appendix A.

**) The factor $\mathbb{N}\left(e^{\alpha}, e^{a}\right), E q$. (22), can be absorbed in the normalization of $\bar{Z}\left(e^{\alpha}, e^{a}\right)$, although it vanishes when zero mass modes are present. If the path integral were extended also over the vielbein $\mathrm{E}^{\mathrm{A}}$, the factor $\mathbb{N}\left(\mathbb{E}^{\mathrm{A}}\right) / \mathbb{N}\left(e^{\alpha}, e^{a}\right)$ should, however, be included in the integrand. 


$$
\sum_{0 \neq j<T} c_{j}^{\prime} \frac{i}{(2 \pi)^{4}} \int \frac{1}{p^{2}+M_{j}^{2}} d^{4} p=\frac{1}{4 \pi^{2}} \sum_{0 \neq j<T} c_{j}^{\prime}\left[-M^{2}+M_{j}^{2} \log \left(1+\frac{M^{2}}{M_{j}^{2}}\right)\right]_{(29)}
$$

The first term $\left(\sim-\mathbb{T}^{2} \mathbb{M}^{2}\right)$ is independent of the background radius $L$ and should be absorbed in the definition of $\bar{\psi} \Psi$, in complete analogy with the procedure commonly adopted. ${ }^{18)}$ in calculations of Casimir energies ${ }^{*}$. The second term is, however, I dependent and physically significant, hence we rewrite the consistency condition (28) in the following form

$$
1=\frac{x L^{-2}}{4 \sqrt{2} \pi^{2}} \sum_{0 \neq j<T} c_{j}^{3} d_{j} \log \left(1+\frac{M^{2} L^{2}}{c_{j}^{2}}\right) .
$$

of course, I cannot yet be computed from (30), unless the cut-off $\left(\mathrm{M}^{2}, \mathrm{~T}\right)$ can be determined by other means. Therefore, the simple model considered here does not quite lead to a numerical prediction for $I$. However, it should be clear that the mechanisms discussed here are quite general and that a consistency condition of the same kind as Eqs. (24) and (25) should be expected to hold also in other multidimensional theories, where spinors are coupled to EC gravity. If one succeeded in finding a model which is ultraviolet finite $\left.{ }^{* *}\right)$, the consistency condition should become cut-off independent and I should consequently be completely calculable from the dynamical

mechanism of spin-torsion compactification. This possibility is, of course, extremely interesting, because $\sqrt{x} / \mathrm{L}$ measures the magnitude of the bare YangMills coupling constant ${ }^{1)-6)}$ after dimensional reduction to $M_{4}^{0}$.

It should also be observed that the term $n=2$ in Eq. (21) leads to a kinetic term and to a mass counter-term for the internal torsion field $\mathrm{K}_{\mathrm{abc}}^{(0)}$. Therefore, this field can in principle acquire a physical mass much lower than the bare mass $\left(\sim x^{-\frac{1}{2}}\right)$.

Other possible physical effects of the spin-spin contact interaction shall be discussed elsewhere, particularly in connection with the proposal made in Ref. 20).

*) One could also modify our approach and keep the leading divergence of $\mathrm{Eq}$. (29), as in the N-JL model. The "wrong" sign $\left(\sim-M^{2}\right)$ might be avoided by exploiting the lack of positivity of $M_{o p}$. We feel that the advocated subtraction is, however, physically bettex motivated.

**) It is possible that a multidimensional theory with local supersymmetry could be finite. 


\section{ACKNOWLEDGEMENTS}

We thank T. Regge and H. Nicolai for stimulating discussions. One of us (C.A.0.) gratefully acknowledges the hospitality and financial support of the CERN Theoretical Physics Divisian.

\section{$* * * * * * *$}

\section{REFERENCES}

1) T. KaIuza, Z.Sitzber.Preuss.Akad.Wiss. 966 (1921);

O. Klein, Z-Phys. 37 (1925) 895;

A. Einstein and P. Bergmann, Ann. Math. 39 (1938) 683.

2) P. Jordan, Astronom. Nachr. 276 (1948) 193;

Y.R. Thiry, Compt.Rend. 226 (1948) 216.

3) Y.M. Cho and P.G.O. Freund, Phys.Rev. D12 (1975) 1711;

C.A. Orzalesi, Fortschr.Phys. 29 (1981) 413, and references therein.

4) E. Cremmer and J. Scherk, Nuclear Phys. B108 (1976) 409;

J.F. Luciani, ibid.B135 (1978) 111.

5) E. Witten, Nuclear Phys. B186 (1981) 412;

A. Salam and J. Strathdee, Ann.Phys. 141 (1982) 316.

6) C. Destri, C.A. Orzalesi and P. Rossi, Ann.Phys. 147 (1983) 321.

7) A. Trautman, Sympos. Mathem. 12 (1973) 139;

F.W. HehI, P. Von der Heyde, G.D. Kerlick and J.M. Nester, Revs.Mod. Phys. 48 (1975) 393.

8) E. Cremmer, B. Julia and J. Scherk, Phys. Letters 76B (1978) 409;

E. Cremmer and B. Julia, Nuclear Phys. B159 (1979) 141.

9) P.G.0. Freund and M.A. Rubin, Phys. Letters 94B (1980) 233;

M.J. Duff and C.N. Pope, in Supersymmetry and Supergravity, Ed. by S. Ferrara, J.G. Taylor and P. van Nieuwenhuizen, World Scientific (1982);

B. Biran, F. Englert, B. de Wit and H. Nicolai, Phys.Letters 124B (1983) 45 .

10) M.J. Duff and C.A. Orzalesi, Phys. Letters 122B (1983) 37.

11) C.A. Orzalesi and M. Pauri, Phys. Letters 107B (1981) 186;

C.A. Orzalesi, in Froceedings of Conf. on Differential Geometric Methods in Theor.Physics, Trieste 1981, Ed. by G. Denardo and H.D. Doebner, World Scientific (1983).

12) C.A. Orzalesi, preprint CERN.TH-3647, to be published. 
13) Y. Nambu and G. Jona-Lasinio, Phys.Rev. 122 (1961) 345.

14) T. Eguchi and H. Sugawara, Phys.Rev. D10 (1974) 4257;

T. Kugo, Progr.Theor.Phys. 55 (1976) 2032;

K. Kikkawa, ibid 56 (1976) 974.

15) A. Jichnerowicz, Compt.Rend.Acad.Sci.Paris 257 (1963) 7 .

16) G.D. Kerlick, Phys.Rev. D12 (1975) 3004;

M. Soffel, B. Muiller and W. Greiner, Phys.Letters 70A (1979) 167.

17) H.B.G. Casimir, Proc.Kon.Ned.Akad.Wet. 51 (1948) 793.

18) B.S. De Witt, Phys. Reports 19 (1975) 295.

19) A. Lichnerowicz, Géométrie des Groupes de Transformation, Dunod (1958).

20) S. Bergia, C.A. Orzalesi and G. Venturi, Phys.Letters 123B (1983) 205. 